\title{
Contribution of extraretinal signals to the scaling of object distance during self-motion
}

\author{
F. PANERAI, V. CORNILLEAU-PÉRÈS, and J. DROULEZ \\ Laboratoire de Physiologie de la Perception et de l'Action, CNRS, Paris, France
}

\begin{abstract}
We investigated the role of extraretinalinformation in the perception of absolute distance. In a computersimulated environment, monocular observers judged the distance of objects positioned at different locations in depth while performing frontoparallel movements of the head. The objects were spheres covered with random dots subtending three different visual angles. Observers viewed the objects at eye level, either in isolation or superimposed on a ground floor. The distance and size of the spheres were covaried to suppress relative size information. Hence, the main cues to distance were the motion parallax and the extraretinal signals. In three experiments, we found evidence that (1) perceived distance is correlated with simulated distance in terms of precision and accuracy, (2) the accuracy in the distance estimate is slightly improved by the presence of a ground-floor surface, (3) the perceived distance is not altered significantly when the visual field size increases, and (4) the absolute distance is estimated correctly during self-motion. Conversely, stationary subjects failed to report absolute distance when they passively observed a moving object producing the same retinal stimulation, unless they could rely on knowledge of the three-dimensional movements.
\end{abstract}

Perceived location of objects in three-dimensional space is of paramount importance for planning and executing actions such as locomotion or grasping. It can be estimated with respect to two reference frames: the egocentricframe, in which the distance of a point in the environment from the subject (i.e., absolute distance) is evaluated, and the exocentric frame, in which the relative distance between two disconnected points in the environment is estimated. To perform egocentric depth estimation, the brain integrates a wide variety of types of visual information (Cutting \& Vishton, 1995; Philbeck, Loomis, \& Beall, 1997) referred to as cues, which under normal circumstances lead to percepts of reasonable, even nearly metric accuracy. Visual cues to depth are often distinguished as optical (i.e., related to the structure of the light entering the eyes) and ocular (i.e., related to the state of the eyes; Palmer, 1999). A number of earlier studies have investigated the extent to which specific cues lead to an absolute or relative characterization of space. Several of these investigations show that ocular cues in near space contribute to absolute distance estimates (Fisher \& Ciuffreda, 1988; Gogel, 1961; Grant, 1942; Wallach \& Floor, 1971). Conversely, optical cues like optic flow ${ }^{1}$ help to specify distance only to a scale factor (Koenderink, 1986; Lee, 1980; Prazdny, 1983). Recent research has examined the

This work was partially supported by Human Frontier Research Grant RG-71/96 B. The authors gratefully acknowledge France Maloumian for her help in preparing the figures, Sid Wiener for his comments on the manuscript, and Mark Wexler for technical assistance. Correspondence should be addressed to F. Panerai, Laboratoire de Physiologie de la Perception et de l'Action, CNRS/Collège de France, 11 pl. Marcelin Berthelot, 75005 Paris, France (e-mail: francesco.panerai@ college-de-france.fr). quantitative combination of depth information from different sources, with major emphasis on the cues available to a stationary observer (Braunstein, Andersen, Rouse, \& Tittle, 1986; Bruno \& Cutting, 1988; Clark \& Yuille, 1990; Landy, Maloney, Johnston, \& Young, 1995; Poom \& Börjesson, 1999). From a computational perspective, besides the use of ocular cues (e.g., accommodation and convergence), the visual system is capable of recovering absolute measurements with the use of two purely visual mechanisms: (1) vertical disparities obtained from binocular viewing providing information equivalent to ocular convergence (B. J. Rogers \& Bradshaw, 1993); and (2) under monocular viewing, quantitative information can be recovered if the horizon is visible or can be surmised from information such as a ground surface (Sedgwick, 1986). Theoretically, a third mechanism could be imagined, if one accounts for the possibility that the visual system is able to calibrate retinal optic flow with extraretinal signals related to selfmotion (Koenderink \& van Doorn, 1975; Simpson, 1993). The term extraretinal is used in the context of this work to indicate sensory signals related to the movement of the observer (vestibular, proprioceptive, efference copies of motor commands, etc.). Recently, a few authors have examined the role of extraretinal (i.e., nonvisual) information in structure-from-motion (SFM) discrimination tasks. The general emerging picture is that extraretinal information contributes to removing ambiguities in extraction of SFM surface parameters, such as curvature or orientation, helping to determine more reliable three-dimensional percepts (Cornilleau-Pérès \& Droulez, 1994; S. Rogers \& B. J. Rogers, 1992; van Damme \& van de Grind, 1996; Wexler, Panerai, Lamouret, \& Droulez, 2001). On the other 
hand, few authors have pointed out a potential involvement of extraretinal signals in the calibration of motion parallax cues to recover absolute distance information (Bingham \& Stassen, 1994; Eriksson, 1973, 1974). In the present investigation, we tested for experimental evidence of the role of extraretinal signals in the estimation of absolute distance. We compared performance of monocular subjects in two conditions: when they actively explored stationary objects while making head movements, and when they passively observed moving objects from a stationary position. The visual motion experienced by the subject in the active condition is reproduced in the passive condition by moving objects on the basis of previously recorded head trajectories. The results show that performance in estimating distance in the two conditions is dramatically different and suggest that extraretinal information plays an important role in scaling motion parallax for estimating object distance. Moreover, we examined the influence of two factors on subject's accuracy in reporting distance-namely, the presence of a ground surface (J. J. Gibson, 1950; Sinai, Ooi, \& He, 1998) and the size amplitude of retinal stimulation (Coello \& Grealy, 1997; B. J. Rogers \& Bradshaw, 1993). According to previous investigators (Dijkstra, Cornilleau-Pérès, Gielen, \& Droulez, 1995), the size of the stimulated visual field can be an effective parameter influencing the perception of three-dimensional shape from motion. Finally, work on visual-vestibular interaction (Dichgans \& Brandt, 1978) suggests that the motion field size could influence the processing and the interpretation of optic flow, eliciting either an object motion or a self-motion percept. Consequently, it would be interesting to test whether the size parameter could also play a role in the context of distance estimation from self-motiongenerated motion parallax.

\section{Head-Generated Retinal Motion and Distance Estimation}

Early investigations concerning motion parallax as a cue to distance perception date back to the work of Bourdon $(1898,1902)$. By using stimuli as simple as spots of light, he investigated the depth order and the relative distance between two points. Further studies (E. Gibson, J. J. Gibson, Smith, \& Flock, 1959), with complex stimuli (i.e., motion parallax with a gradient of velocities) replacing the simple spots of light, have led to similar results, suggesting that motion parallax is a rich source of information for discriminating relative distance, but not for estimating absolute distance. In later studies, the importance of head movement in the perception of absolute distance was proposed by Park (1964) and by Dees (1966). This notion was further revised by Ferris (1972) and Johansson (1973), with Johansson, in particular, demonstrating the first convincing evidence of the importance of the simultaneous occurrence of visual and kinesthetic cues derived from head movements. Johansson observed that under monocular vision, subjects who were actively moving their heads reported veridical perception of absolute distance within a range defined as near space $(<2 \mathrm{~m})$. This result, though, diverged from other contemporary findings, such as those of Gogel and Tietz (1973), who observed a high variability in accuracy of distance estimation when a subject explored objects while making head movements in reduced viewing conditions, such as a single light spot in a dark surround (see also Gogel, 1982; Gogel \& Tietz, 1979).

Later studies (Braunstein, 1976; B. Rogers \& Graham, 1979) fully uncovered the extent to which head-generated and passively experienced motion parallax convey relative depth and three-dimensional structure information.

Recently, the issue of the use of head movements as a means to estimate absolute distance has resurged into focus. Studies of animal behavior (Collett, 1978; Collett \& Harkness, 1982; Ellard, Goodale, MacLaren Scorfield, \& Lawrence, 1986; Ellard, Goodale, \& Timney, 1984; Goodale, Ellard, \& Booth, 1990) as well as of enucleated patients have indicated that head movement plays a prominent role in motor tasks requiring absolute distance information (Marotta, Kruyer, \& Goodale, 1998; Marotta, Perrot, Nicolle, Servos, \& Goodale, 1995). In the Ellard et al. (1986; Ellard et al., 1984) and Goodale et al. (1990) studies, for example, Mongolian gerbils spontaneously performed vertical head movements just before jumping across a gap. The authors observed a strong correlation between the amplitude of the animal's head bobs and the gap length, which suggests a tendency to rely on motion parallax to estimate large distances. Moreover, since jumping is a ballistic motor act, precision in estimating distances is required. In a different context, Marotta et al. (1995) showed that enucleated patients, when engaged in grasping behavior, performed wide and fast head movements. The authors' interpretation of this is that the patients used horizontal and vertical head movements to enhance retinal motion cues for distance computation (see also Gonzalez, Steinbach, Ono, \& Wolf, 1989; Steinbach, Ono, \& Wolf, 1991). Further studies on reaching under monocular viewing conditions by Bingham and Stassen (1994) confirmed the finding that head movements in the direction of the target facilitate the recovery of its absolute distance (Pagano \& Bingham, 1998). However, these results are specific for reaching space and, in some cases, refer to experimental conditions in which other types of sensory information were available, such as haptic feedback (Wickelgren, McConnell, \& Bingham, 2000). Our study extends the investigation well beyond reaching space and was conducted with standing subjects performing head movements of larger amplitudes in unrestrained conditions.

More precisely, in the present work, we questioned the contribution of extraretinal signals to the scaling of optical information during head motion when motion parallax was the only optical cue available. Are extraretinal signals used at all to calibrate retinal motion cues? If the answer is positive, does this calibration enable an active 


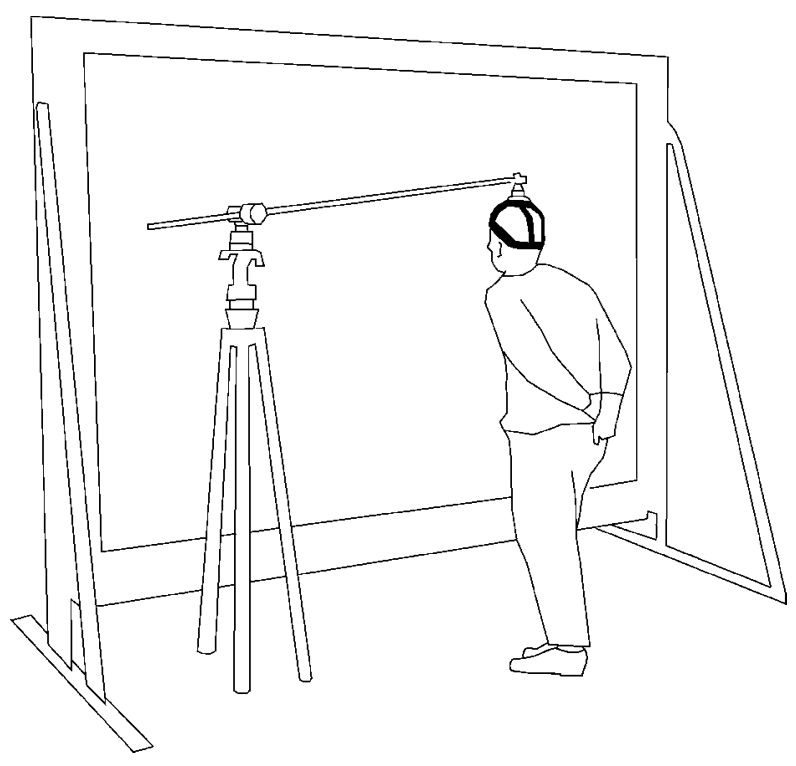

Figure 1. The experimental apparatus.

observer to recover definite metric distance? We used a computer-simulated environment, consisting of a large field display facility and a head tracker (Panerai, Hanneton, Droulez, \& Cornilleau-Pérès, 1999) to update in real time the appearance of objects in a simple visual scene. The subjects made repetitive frontoparallel head movements while exploring spheres positioned at several different apparent locations in depth and that were textured with random dots. All of the objects were viewed at eye level, in isolation or superimposed over a tiled ground floor. The distance and the size of the object were covaried to suppress relative size information. Hence, the only available distance cues were provided by retinal optic flow and by self-motion information. In Experiment 1, we established the relationship between perceived and simulated distances, probing the influence of the size of the visual field and the presence or absence of the ground floor surface on precision and accuracy of reported distance. In Experiment 2, we compared the performance of subjects in the following two conditions: (1) a self-motion (SM) condition, in which the subjects explored the visual scene with frontoparallel head movements of constant amplitude and (2) an object-motion $(\mathrm{OM})$ condition, in which the subjects experienced the same retinal motion cues, which were this time produced by three-dimensional movements of the object. Finally, in Experiment 3, we compared subject performance in SM and OM conditions when the amplitude of the head movement changed from trial to trial.

\section{EXPERIMENT 1}

Experiment 1 was devised to relate perceived distances and simulated distances in monocular observers explor- ing a visual scene with active head movements. The subjects had to estimate the absolute distance to a sphere presented at eye level while they performed fronto-parallel head movements of natural amplitude and constant frequency. For each successive stimulus presentation, the simulated size and distance of the spheres were covaried in order to eliminate apparent size cues. Therefore, in such controlled conditions, the main cues to distance were retinal motion and extraretinal signals originating from self-motion. In this experiment, we were aiming also at probing the role of visual field size and visual context by presenting spheres that subtended three different angles $\left(6^{\circ}, 15^{\circ}\right.$, and $30^{\circ}$ radius $)$ and by introducing a regularly textured horizontal ground floor.

\section{Method}

Subjects. Nine subjects with normal vision participated in the experiment. None had prior knowledge of the experimental aims or protocol. All of them passed a screening test to assess their capability for distance estimation in the presence of full binocular cues. All were paid for their participation.

Apparatus. The experimental apparatus is pictured in Figure 1. The setup was composed of (1) a large-field screen $(2.43 \mathrm{~m}$ wide $\times$ $1.89 \mathrm{~m}$ tall $),(2)$ a high resolution $(1,280 \times 1,024)$ Barco 800 video projector displaying the visual stimuli on the large screen, (3) an optomechanical tracker with high accuracy (better than $1 \mathrm{~mm}$ ) and low latency (less than $1 \mathrm{msec}$; Panerai et al., 1999) providing data of the position of the subject's eye in real-time, and (4) a PC graphic station (Pentium II, $600 \mathrm{MHz}$ ) with a 3-D graphic engine generating stimuli at a frequency of $85 \mathrm{~Hz}$ based on instantaneous eye position.

Task. On each trial, a sphere appeared at 1 of 12 possible simulated distances from the observer (from 30 to $316 \mathrm{~cm}$, with positions separated by $26 \mathrm{~cm}$ ). The object was viewed at eye level either in isolation or superimposed onto a horizontal textured plane. When the stimulus disappeared, a text showing four alternative distance categories was displayed. The subject was instructed to place the perceived distance into one of the four categories. Each object distance was repeated 10 times. In separate sessions, stimuli of three different angular sizes were presented.

Stimuli. The objects were spheres textured with random dots. The number of visible dots was the same throughout trials $(200 \pm 10)$ and was obtained from a constant dot density on the surface of the spheres $\left(1 / \mathrm{cm}^{2}\right.$ for the object at $\left.30 \mathrm{~cm}\right)$. The angular size of the objects was kept constant by covarying the simulated distance and size (see Figure 2). The objects of different angular size $\left(6^{\circ}, 15^{\circ}, 30^{\circ}\right)$ had approximately the same number of points distributed over the surface. Small-size $\left(6^{\circ}\right)$, medium-size $\left(15^{\circ}\right)$, and wide-size $\left(30^{\circ}\right)$ stimuli were presented in three separate experimental sessions. The ground surface was textured with regular squared tiles $(12 \times 12$ tiles, each measuring $30 \times 30 \mathrm{~cm}$ ), beginning at the bottom border of the screen (see Figure 3). The stimulus luminance was in the range of $0.2-0.3 \mathrm{~cd} / \mathrm{m}^{2}$ for the sphere and $0.04-0.08 \mathrm{~cd} / \mathrm{m}^{2}$ for the ground floor surface. The background luminance was in the range of $0.002-0.003 \mathrm{~cd} / \mathrm{m}^{2}$.

Design. A 2 (visual context: OBJ_ISO, OBJ_PLA) $\times 12$ (object distance: $\mathrm{D}=30,56,82,108,134,160,186,212,238,264,290$, $316 \mathrm{~cm}$ ) within-subjects design was used. The design was repeated in three separate experimental sessions for the three angular sizes $\left(6^{\circ}, 15^{\circ}, 30^{\circ}\right)$ of the spherical object.

Screening procedure. At the beginning of the experiment, the subjects underwent a screening in order to assess their ability to recover metric distances in the full-cue condition. Under binocular viewing, the subjects were asked to report the distance in centimeters of several markers placed at different positions in the room. The 


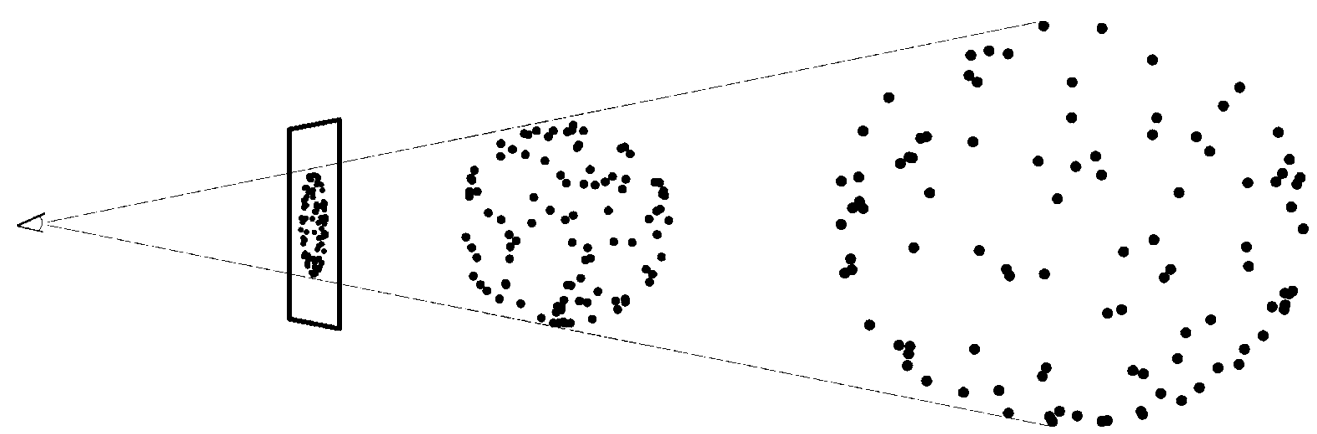

Figure 2. Schematic representation of the stimulus characteristics. The angular size of the stimulus was kept constant by covarying the simulated distance and size of the objects.

markers were numbered sequentially (1-8) and were referenced in an order that avoided judgment of distance by comparison (the range of distances varied from $100-500 \mathrm{~cm}$ ). Each subject was informed when his or her judgments were inappropriate. The procedure was repeated three times with the subject occupying different positions in the room. If the average error was greater than $40 \mathrm{~cm}$, the subject was excluded from the experiment. According to this criterion, only 1 subject was dismissed.

Experimental procedure. Three experimental sessions were conducted employing spheres with the three angular sizes $\left(6^{\circ}, 15^{\circ}\right.$, and $30^{\circ}$ ). The subject had his or her nondominant eye covered. He or she stood facing the horizontal midpoint of the screen at a distance of $60 \mathrm{~cm}$. The stimulus was presented (stimulus time $=7 \mathrm{sec}$ ), and the subject started performing repetitive oscillatory lateral head movements of natural amplitude and oscillation frequency. In order to help maintain the same oscillation frequency throughout trials, the computer played a sound, once a given distance from the initial position was attained, in both directions of movement. At the end of the trial, the visual stimulus disappeared and a dialog with four alternative choices appeared. The subject was then asked to report verbally on the estimated distance of the closer edge of the spherical surface by choosing one of four distance intervals (below $50 \mathrm{~cm}$, between 50 and $100 \mathrm{~cm}$, between 100 and $200 \mathrm{~cm}$, more than $200 \mathrm{~cm}$ ). During each trial, head movements were recorded.

Data analysis. The response data were used to derive for each subject three psychometric curves corresponding to the three distance thresholds $(50,100,200 \mathrm{~cm})$ defined in the interval of simulated distances $(30-316 \mathrm{~cm})$. The curves enable us to establish the correspondence between the simulated distance and the subject's perceived distance at threshold values (the values identifying this correspondence will be referred to with the symbols d50, d100, d200).

To derive a given curve (e.g., d100), the proportion of correct responses in estimating that the object distance exceeded a given threshold (e.g., $100 \mathrm{~cm}$ ) was computed according to the following procedure: The subjects' responses were organized into a $4 \times 12$ matrix (4 responses, 12 simulated distances). The number of repetitions of the stimulus (i.e., 10) was used to normalize the number of responses in each matrix cell. Therefore, each column of the matrix describes the distribution of probabilities (i.e., $p_{1}, p_{2}, p_{3}, p_{4}$ ) of

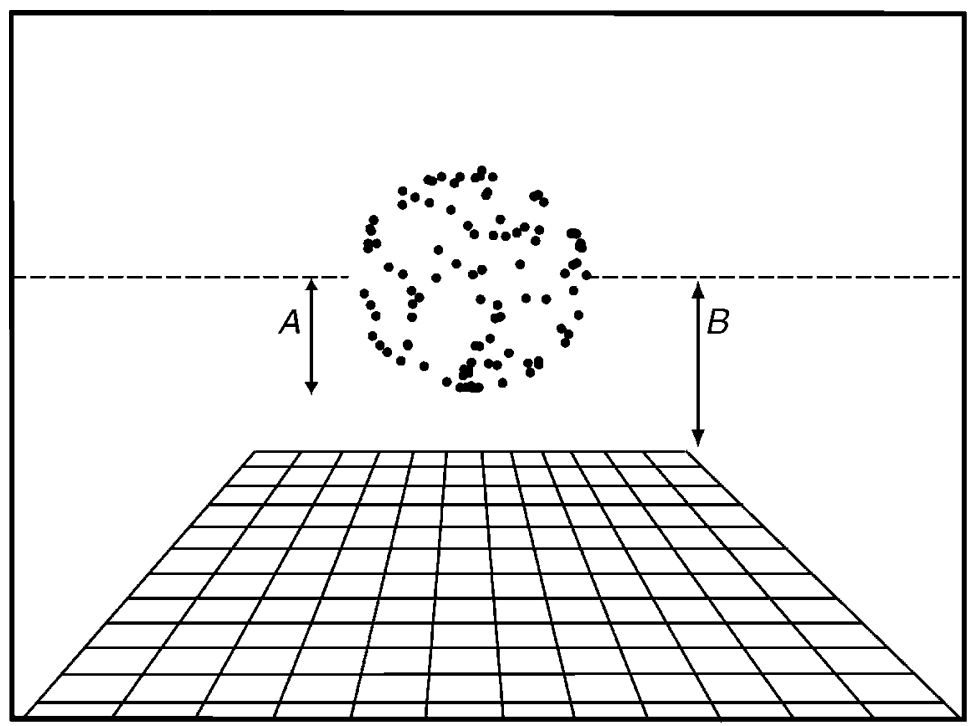

Figure 3. Schematic representation of a frame of an actual display with ground tiles. The parameters $A$ and $B$ indicate, respectively, the angular extension of the sphere (e.g., $A=15^{\circ}$ ) and the height from the horizon of the farthest border of the ground tiles' surface $\left(B=20^{\circ}\right.$ approximately $)$. 
the 4-AFC responses, with $p_{1}+p_{2}+p_{3}+p_{4}=1$. In order to compute the proportion of responses for a given distance threshold (e.g., d100) as a function of the simulated distance, response probabilities were cumulated with respect to the threshold value. For the 100 -cm distance threshold, $P(>\mathrm{d} 100)=p_{3}+p_{4}$. For the other two distance thresholds, $P(>\mathrm{d} 50)=p_{2}+p_{3}+p_{4}$ and $P(>\mathrm{d} 200)=p_{4}$.

Finally, normal cumulative distribution functions were fitted to the proportion of responses for a particular threshold computed as a function of the simulated distances. The statistical accuracy of the threshold estimates was verified using a bootstrap method (Foster $\&$ Bischof, 1997). Biases and slopes of the psychometric curves were used to derive the estimates of the perceived distance and the variability of perceived distance. Since, for some of the subjects, the data appeared to be incomplete, a criterion was used to decide whether to perform the fitting or not: The proportion of responses for the given distance threshold (i.e., d100) should be characterized by at least one value above the .5 level. If this was the case, the fitting was performed, and the bias and slope were derived. The application of this criterion enabled us to derive a complete set of biases and slopes for only 4 of the 9 subjects. The psychometric curves for these 4 subjects are illustrated in Figure 4. In the Results section, the data is analyzed for each subject, separately.

\section{Results}

Of the 9 subjects, only 4 provided a distribution of responses from which we were able to derive the complete set of the three distance thresholds (i.e., d50, d100, d200) across all experimental conditions. Therefore we analyze their responses first. The bias and the slope of each subject's psychometric curve were entered into two separate repeated measures analyses of variance (ANOVAs). The ANOVA of the bias parameter (3: distance threshold, 50, $100,200 \mathrm{~cm} \times 3$ : angular size, $6^{\circ}, 15^{\circ}, 30^{\circ} \times 2$ : visual context, OBJ_ISO, OBJ_PLA) revealed a significant main effect of distance $[F(2,6)=142.49, p<.05]$ and an interaction of distance and angular size $[F(4,12)=3.57$, $p<.05]$. The ANOVA of the slope parameter (slope indicates variability of perceived distance) revealed only a significant main effect of distance $[F(2,6)=18.04, p<$ $.05]$. In both cases, we observed the absence of a main effect of angular size and of visual context. The analysis was extended to the remaining 5 subjects, with eight missing points (five for the threshold of $200 \mathrm{~cm}$, and three for the threshold of $100 \mathrm{~cm}$ ), confirming the previous findings. In Figure 5, the averaged perceived distance and variability of perceived distance for the 9 subjects are shown. On the right side of Figure 5, note that the curve representing variability of perceived distance in the presence of the ground surface (OBJ_PLA) is below
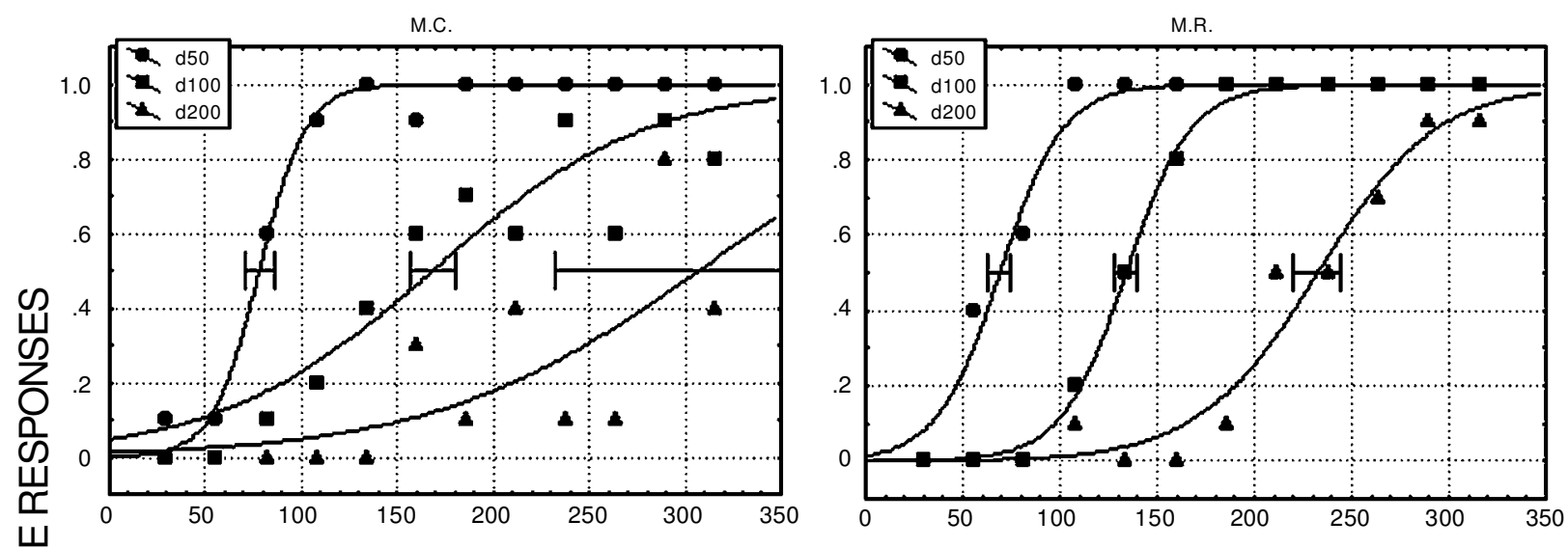

岁
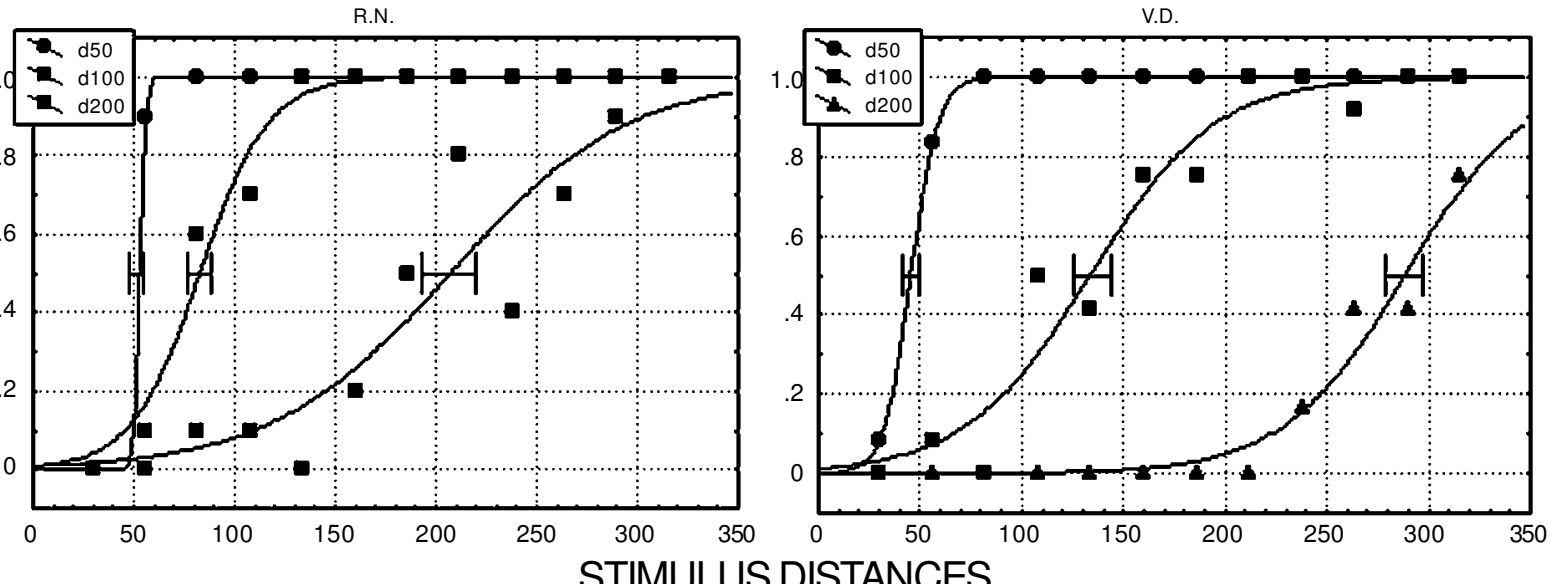

Figure 4. A sample of the psychometric curves derived from the 4-AFC data. The four panels refer to the 4 subjects that gave a complete set of data. They were obtained from the data corresponding to virtual objects of $15^{\circ}$ angular size. Ordinates: proportion of responses for the corresponding distance threshold (e.g., d50, d100, d200). Abscissae: stimulus distance. 

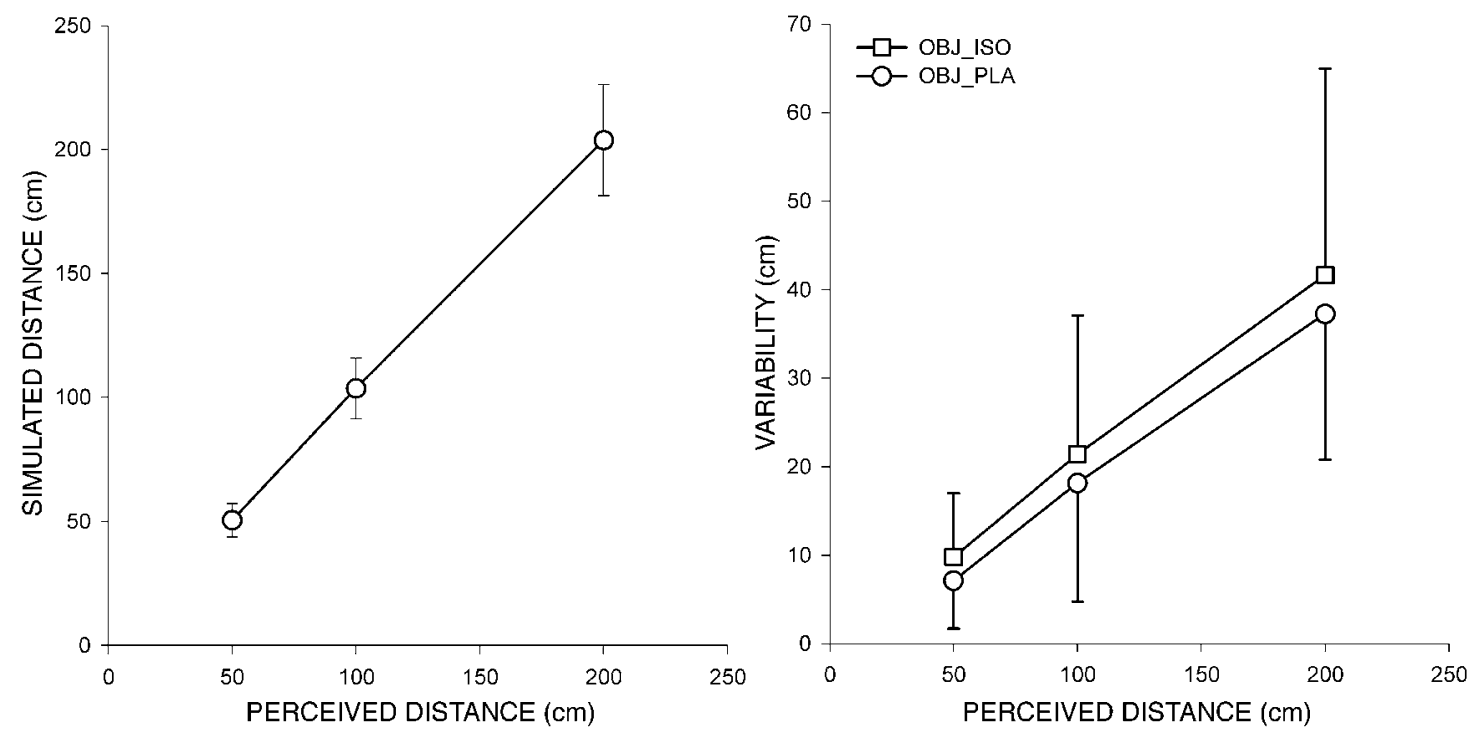

Figure 5. Perceived distance and variability of perceived distance. Left: mean values of the simulated distances (ordinates) corresponding to the three thresholds of perceived distance $(50,100$, and $200 \mathrm{~cm})$ on the abscissae. Right: variability of perceived distance for each distance threshold.

the OBJ_ISO curve. Therefore, the presence of a ground surface tended to decrease the variability of responses.

Consistent with the psychophysical responses, almost all the subjects reported verbally that in the presence of the ground surface, the object was located "more easily." This systematic observation led us to perform an analysis of the responses of the 9 subjects in terms of object visual context (i.e., the object isolated, OBJ_ISO vs. the object su- perimposed onto the ground surface, OBJ_PLA). The rationale for grouping the subjects' responses for the three different angular sizes was the absence of a significant main effect for this factor. In Figure 6, each point represents data from 1 subject: respectively, the threshold (left) and the variability of threshold of perceived distance (right) obtained in the two conditions (OBJ_ISO, the abscissae vs. OBJ_PLA, the ordinates). The regres-
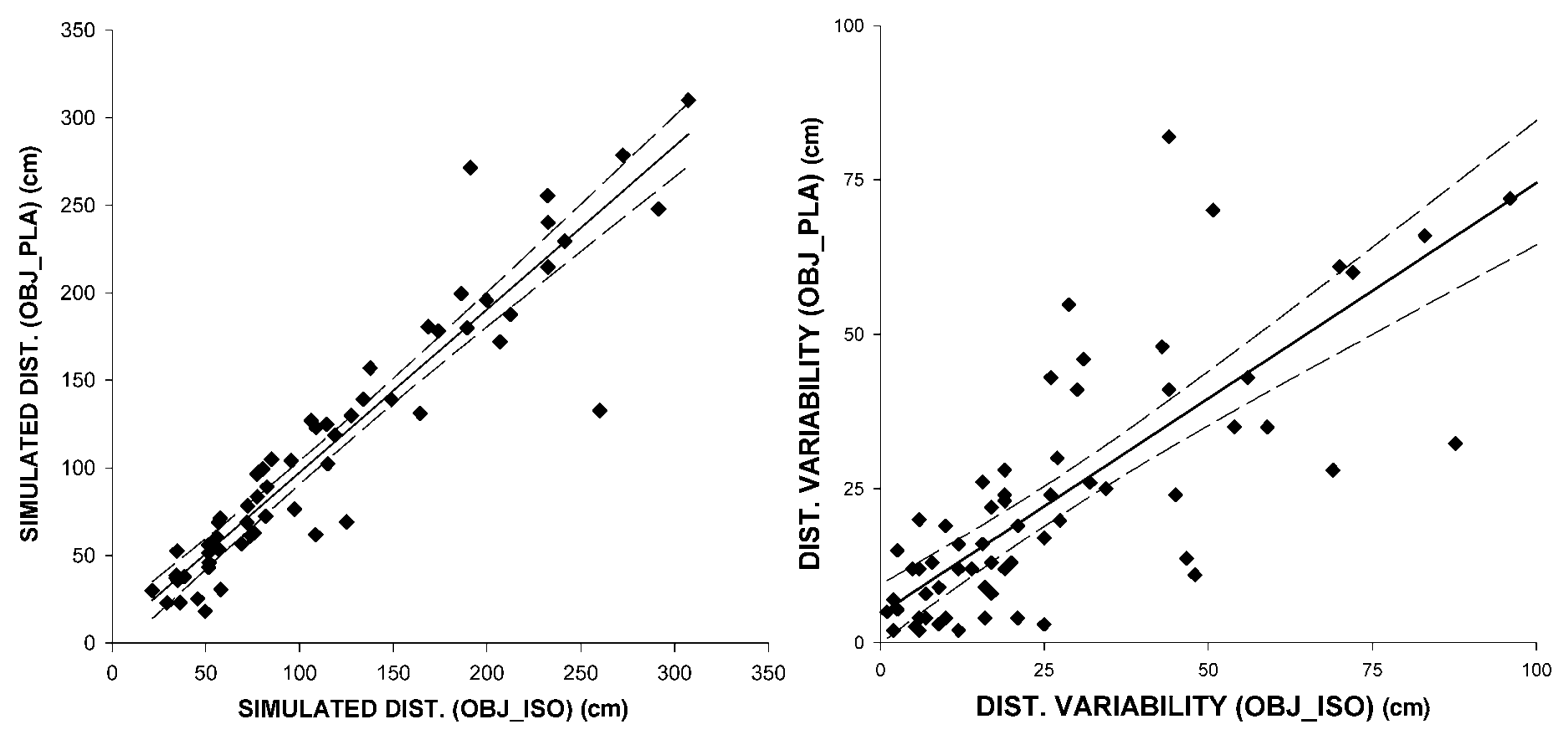

Figure 6. Regression analysis for the perceived distance and variability of perceived distance as function of the visual context (OBJ_ISO vs. OBJ_PLA). The abscissa represents data for the object presented in isolation (OBJ_ISO). On the ordinate axis, data correspond to objects superimposed on the ground surface (OBJ_PLA). The points correspond to the complete (i.e., 4 subjects) and incomplete (i.e., remaining 5 subjects) data set obtained in both conditions of Experiment 1. Dashed lines represent graphically the confidence intervals. 
Table 1

Regression Analysis Parameters of Perceived Distance Responses Grouped Over the Three Different Angular Sizes

\begin{tabular}{lccc}
\hline \multicolumn{1}{c}{ Regression Data } & Slope & 95\% Confidence Interval & $r^{2}$ \\
\hline Perceived distance & $0.93 \pm 0.04$ & 0.84 to 1.02 & .88 \\
Variability of perceived distance & $0.71 \pm 0.09$ & 0.54 to 0.89 & .59 \\
\hline
\end{tabular}

sion line for the two sets of data was derived, and Table 1 summarizes the values of the slopes and the confidence intervals.

The regression coefficients indicate that the variability of perceived distance is significantly reduced in the presence of a ground floor (the slope of the regression line is smaller than 1 , as indicated on the second line of Table 1). However, the ground surface introduces no significant change in the perceived distance, since the slope of the regression line is not significantly different from 1. Finally, for all of the 9 subjects, verbal reports were collected at the end of Experiment 1. All the subjects indicated that for close enough objects (i.e., 30 and $56 \mathrm{~cm}$ ), they had the vivid impression that they could easily reach them. The verbal reports were qualitatively compatible with the values obtained from the psychometric curves.

Subjects' head movements. The subjects moved their heads along sinusoidal trajectories in the frontoparallel plane. Trajectories were recorded and processed on a trial-by-trial basis. Root mean square amplitude (RMS) was derived for each sinusoidal head trajectory, and the average RMS amplitude was calculated for each distance value. A 2 (visual context: OBJ_ISO, OBJ_PLA) $\times 12$ (distance: $30,56,82,108,134,160,186,212,238,264$, $290,316 \mathrm{~cm}) \times 3$ (angular size: $\left.6^{\circ}, 15^{\circ}, 30^{\circ}\right)$ repeated measures ANOVA was performed on the data from 7 of the 9 subjects (for 2 of the subjects, the head trajectory files had not been stored in the computer). The ANOVA revealed a significant main effect of distance $[F(11,44)=$ $3.27, p<.05]$ and a significant interaction of distance and angular size $[F(22,88)=4.24, p<.05]$. Figure 7 summarizes these results, showing that head movements tended to be smaller for the short distance values $(30-50 \mathrm{~cm})$, then increased for intermediate distances $(50-200 \mathrm{~cm})$, slightly decreasing at larger distances $(>200 \mathrm{~cm})$. In spite of this significant trend, the maximum change of amplitude across distances represents only about $16 \%$ of the amplitude, and half of the standard deviation. Therefore we can consider that the changes in the amplitude of head movement with distance should only affect our results in a minor fashion.

\section{EXPERIMENT 2}

In the previous experiment, we found that the subjects' reports of absolute distance were highly correlated with simulated distance. The purpose of Experiment 2 was

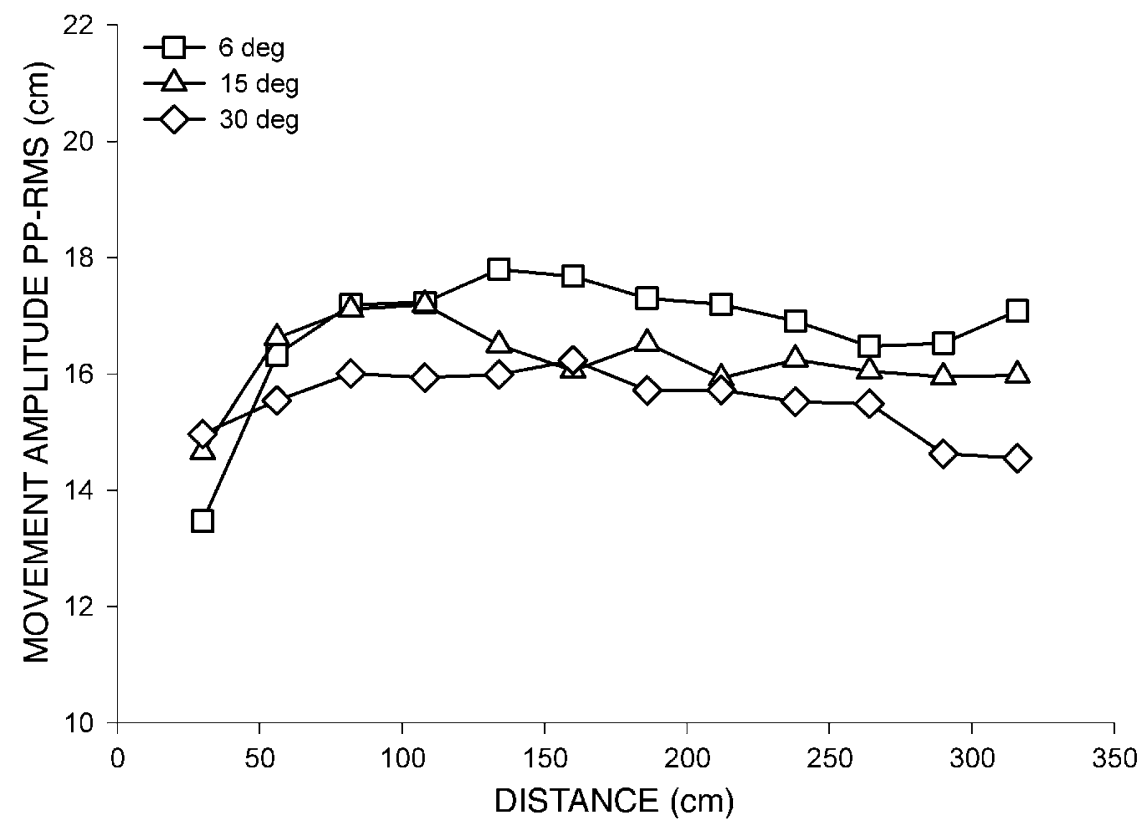

Figure 7. Amplitude of natural head movement versus object distance. The amplitudes of head movements (RMS) are shown as a function of object distance $(30-316 \mathrm{~cm})$ for the three different object angular sizes $\left(6^{\circ}, 15^{\circ}\right.$, and $\left.30^{\circ}\right)$. 


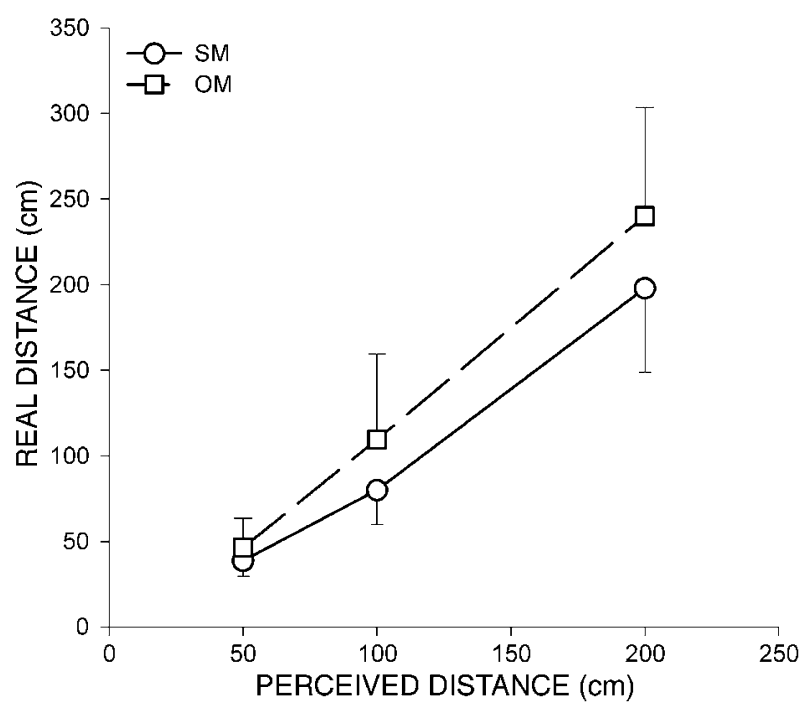

Figure 8. Perceived distance in the $S M$ and $O M$ conditions. OM (squares) and SM (circles) curves indicate the average threshold among 10 subjects, as obtained for each distance on the abscissa. The standard deviations are plotted in brackets.

to test whether self-motion helps distance estimation. This was done by comparing perceived distance in the two following complementary conditions: (1) the subject experienced retinal motion cues in simultaneous occurrence of extraretinal signals due to self-motion (SM condition), and (2) the same visual motion was observed by a stationary subject, the so-called OM condition. In the latter case, moving the object in three-dimensional space on the basis of previously recorded SM trajectories provided an identical head-centered optical stimulation. Therefore, from a sensory point of view, the subjects in the SM and $\mathrm{OM}$ conditions experienced the same optic array.

\section{Method}

Subjects. Ten individuals between the ages of 18 and 35 years served as subjects. All reported normal or corrected-to-norm al vision. All were naive and were paid on an hourly basis for their participation

Apparatus. The apparatus was the same as that in Experiment 1.

Task. The task was the same as that in Experiment 1. In the OM condition, the subjects observed from a stationary position the object moving along a 3-D trajectory. The trajectory was generated on the basis of the head-position data recorded in the previous SM condition.

Stimuli. The medium size $\left(15^{\circ}\right)$ stimuli of Experiment 1 were used, in the absence of a tiled ground floor surface. In the OM trials, the subjects experienced an identical head-centered optical stimulation, without head movement. Each OM trial corresponded to a preceding SM trial, in that the virtual object was identical and the relative motion between the subject's eye and the virtual object were the same in OM and SM trials. During an SM trial, let $r_{0}$ indicate initial position, and $r$ the eye position at a given moment (i.e., relative to the center of the stationary virtual object $O_{0}$ ). At the corresponding moment during the $\mathrm{OM}$ trial, the center of the virtual object $O$ was moved from its initial position $\left(O_{0}\right)$ by an amount resulting from the following:

$$
O=O_{0}-\left(r-r_{0}\right)
$$

Therefore, in a head-centered reference frame, the subject experiences an identical optical stimulation.

Design. A 2 (movement condition: SM, OM) $\times 12$ (object distance: $D=30,56,82,108,134,160,186,212,238,264,290$, $316 \mathrm{~cm}$ ) within-subjects design was used.

Procedure. At the beginning of the experimental session, each subject performed 36 trials in order to get acquainted with the large field display $(189 \times 243 \mathrm{~cm})$ and the movement constraints (smooth, repetitive, frontoparallel head oscillations). On each trial, the subject occupied the same initial position, facing the horizontal midpoint of the screen at a distance of $60 \mathrm{~cm}$. The stimulus was displayed at eye level for four cycles of movements, after which a text indicating the four possible answers was presented on the screen. The subjects responded via a joystick, indicating one of the four alternative choices.

Data analysis. The same analysis was performed as that described for Experiment 1.

\section{Results}

All 9 subjects provided a distribution of responses from which we were able to derive the three distance thresholds across all experimental conditions. A 2 (condition: SM, OM) $\times 3$ (distance: $50,100,200)$ repeated measures ANOVA was conducted on the bias of the subjects' psychometric curves. The ANOVA revealed a significant main effect of condition $[F(1,9)=9.56, p<.05]$, a large effect of distance $[F(2,18)=141.46, p<.05]$, and a significant condition $\times$ distance interaction $[F(2,18)=$ $7.01, p<.05]$. Figure 8 graphically displays these results. It is interesting to note that in the OM condition (dashed line), distances were underestimated relative to those of the corresponding SM condition. This underestimation error increased with distance $(100$ and $200 \mathrm{~cm})$. A Wilcoxon matched pair test run on the two larger distances confirmed the significant two-way interaction found by the ANOVA and revealed a significant difference between the OM and SM conditions $(D=100 \mathrm{~cm}$, mean difference $=20.35 \mathrm{~cm}, p<.05$ and $D=200 \mathrm{~cm}$, mean difference $=27.94 \mathrm{~cm}, p<.05$ ).

As for the variability of the distance estimate obtained from the inverse slope of the subjects psychometric curves, a 2 (condition: $\mathrm{SM}, \mathrm{OM}) \times 3$ (distance thresholds: $50,100,200 \mathrm{~cm})$ repeated measures ANOVA revealed a

Table 2

Head Movement Parameters

\begin{tabular}{ccc}
\hline \multirow{3}{*}{$\begin{array}{c}\text { Object } \\
\text { Distance }\end{array}$} & $M$ & $S D$ \\
\cline { 2 - 3 } Amplitude \\
56 & 14.3 & 4.8 \\
82 & 16.2 & 4.9 \\
108 & 16.8 & 4.9 \\
134 & 16.8 & 5.2 \\
160 & 16.8 & 5.1 \\
186 & 16.6 & 5.2 \\
212 & 16.5 & 4.8 \\
238 & 16.3 & 5.1 \\
264 & 16.2 & 5.1 \\
290 & 16.0 & 4.9 \\
316 & 15.9 & 4.9 \\
\hline
\end{tabular}

Note-Distance measured in centimeters. 


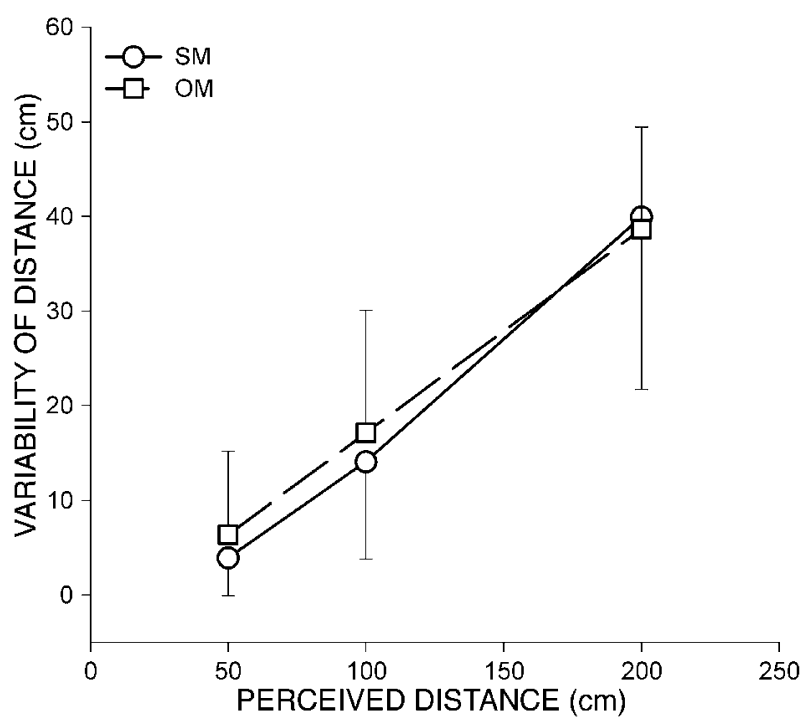

Figure 9. Variability of perceived distance (SM vs. OM) as a function of the actual distance. The average slope of the psychometric curves obtained for the 10 subjects is indicated together with their standard deviations.

significant main effect only for distance $[F(2,18)=$ $43.48, p<.05$ ] (see Figure 9, right). On the other hand, no significant effect of condition $[F(1,9)=0.66, p<.43]$ and no condition $\times$ distance interaction $[F(2,18)=0.46$, $p<.63$ ] were found.

\section{EXPERIMENT 3}

Experiment 2 showed that perceived distance in the OM condition was not far from that in the SM condition. A possible interpretation is that the subjects assumed a constant 3-D displacement of the objects in the OM condition and used this assumption to recover the absolute distance through the apparent motion (the larger the motion, the closer the object). In Experiment 3, we tested this hypothesis by having the subjects perform exploratory movements of different amplitudes. According to our hypothesis, the subjects in the SM condition should be able to judge distance correctly because they rely on extraretinal information to scale the retinal motion cues. On the other hand, due to the lack of extraretinal signals, the subjects in the OM condition should fail to estimate the object distance and will perceive as farthest the stimuli having the smallest optic flow (or ratio $A / D, A$ being the motion amplitude and $D$ the distance).

\section{Method}

Subjects. Nine individuals between the ages of 20 and 35 years served as subjects. They all had normal or corrected-to-normal vision. All were naive and performed in both the SM and OM conditions, being paid on an hourly basis.

Apparatus. The apparatus was the same as that in Experiment 1.

Task. On each trial, an object was displayed at one of three possible simulated distances $(D=50,100,150 \mathrm{~cm})$. The task was to in- dicate the perceived distance as belonging to one of two intervals (closer than $1 \mathrm{~m}$, farther than $1 \mathrm{~m}$ ), following a 2-AFC scheme.

Stimuli. The same stimuli as in Experiment 2 were used here.

Design. A 3 (object distance: $D=50,100,150 \mathrm{~cm}) \times 3$ (movement amplitude: $A=5,10,15 \mathrm{~cm}) \times 2$ (movement condition: selfmotion vs. object motion) within-subjects design was used. For both OM and SM conditions, an identical optical stimulation was experienced by the subjects in those trials in which the movement amplitude and the object distance matched in proportional pairs (i.e., $A / D=5 / 50,10 / 100,15 / 150$ ). The distance and the movement amplitude were randomly chosen. Each distance was repeated 10 times.

Procedure. Prior to the beginning of the experiment, each subject performed 36 trials in order to get acquainted with the large field display and the three different movement amplitudes $(A=5$, $10,15 \mathrm{~cm})$. The subjects were instructed to pay attention to sounds generated by the computer that indicated the spatial limits (i.e., amplitude) for the current movement. During each trial, the amplitude and the frequency of the movement were monitored in real time by the computer, and an incorrect performance determined the restart of the current trial. In Table 3, we summarize the data of head movement recorded during the experiment and averaged over all subjects. The head movements were performed in the frontoparallel plane over four cycles. During the first cycle, the subject viewed only a fixation point. For the remaining three cycles, the visual stimulus was present, after which a text indicating two possible distance intervals (i.e., smaller or larger than $100 \mathrm{~cm}$ ) was displayed. The subjects gave their responses via a joystick.

\section{Results}

Figure 10 (left) plots the subjects' responses "perceived distance closer than $1 \mathrm{~m}$ " for trials in which the ratio between the amplitude of movement and the object distance was constant (constant parallax trials, $A / D=$ const). The dark gray bars represent responses from the subjects performing in the SM condition; the light gray bars represent responses from the subjects performing in the OM condition. A three-way (distance: $50,100,150 \mathrm{~cm}$ ) repeated measures ANOVA performed on the SM responses revealed a significant main effect of distance $[F(2,16)=39.09, p<.05]$. The same analysis performed on the OM responses revealed no significant effect. Moreover, responses in the OM condition were not significantly different from chance level (Wilcoxon test, $D=50 \mathrm{~cm}, p=.8 ; D=100 \mathrm{~cm}, p=.1 ; D=150 \mathrm{~cm}, p=$ $.3)$, but the same test revealed a significant difference for proportion correct responses (i.e., $\mathrm{SM}$ vs. OM) at the two boundaries of the distance interval $(D=50 \mathrm{~cm}, p=.017$; $D=150 \mathrm{~cm}, p=.017)$.

Table 3

Means and Standard Deviations of the Head Movement Amplitudes (RMS) and Frequencies of Oscillation

\begin{tabular}{cccccc}
\hline \multirow{2}{*}{$\begin{array}{c}\text { Movement } \\
\text { Amplitude }\end{array}$} & \multicolumn{2}{c}{ RMS } & & \multicolumn{2}{c}{ FREQ } \\
\cline { 2 - 4 } \cline { 5 - 6 } & $M$ & $S D$ & & $M$ & $S D$ \\
\hline 5 & 5.6 & 1.5 & 0.34 & 0.04 \\
10 & 11.5 & 1.6 & 0.32 & 0.04 \\
15 & 16.8 & 1.9 & 0.31 & 0.05 \\
\hline
\end{tabular}

Note-All measurements are expressed respectively in centimeters and $\mathrm{Hz}$. 

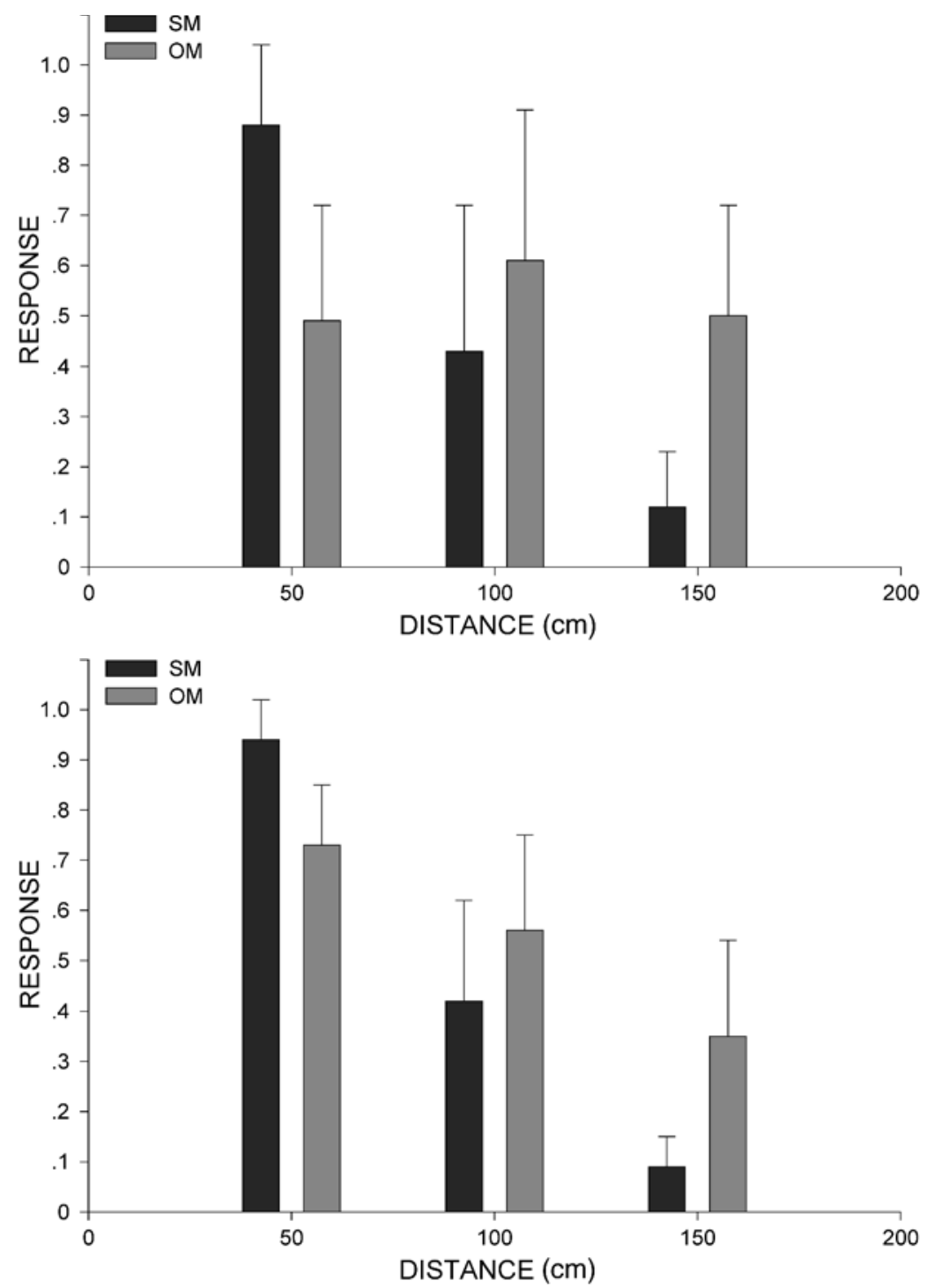

Figure 10. Subjects' responses in the $S M$ and $O M$ conditions. Ordinates represent proportion correct responses $<100 \mathrm{~cm}$. Top: constant ratio trials. Bottom: all trials.

Figure 10 (right) represents the subjects' responses ("perceived distance closer than $1 \mathrm{~m}$ ") for all trials, grouped together. A 2 (condition: $\mathrm{SM}, \mathrm{OM}) \times 3$ (distance: $50,100,150 \mathrm{~cm})$ repeated measures ANOVA revealed a significant main effect of distance $[F(2,16)=$ $191.81, p<.05]$ and a significant condition $\times$ distance interaction $[F(2,16)=12.38, p<.05]$. Both response distributions decrease monotonically. Note that the difference in the $\mathrm{OM}$ responses for the constant parallax trials versus all trials, suggests that the stationary observer makes the assumption that the 3-D object movement is constant. When this assumption was wrong (i.e., for constant parallax trials), the subjects failed to recover the object's distance (compare the OM bars in Figure 10, right, and Figure 10, left). On the other hand, the subjects perform- ing in the SM condition were able to give accurate responses when the ratio $A / D$ was constant (compare now the SM bars in Figure 10, right, and Figure 10, left).

Further insights are provided from the analyses of the proportion correct responses for objects positioned at a distance of $100 \mathrm{~cm}$. Figure 11 (left) plots responses against the amplitude of subject movement $(5,10,15 \mathrm{~cm})$. The dark bars represent responses given in the SM condition; the light bars represent responses given in the OM condition (here the bars represent "perceived distance larger than $100 \mathrm{~cm}$ "). A 2 (condition: SM, OM) $\times 3$ (movement amplitude: $5,10,15 \mathrm{~cm}$ ) repeated measures ANOVA revealed a significant main effect of movement amplitude $[F(2,16)=10.83, p<.05]$. A regression analysis performed on the SM and OM responses (see Figure 11) re- 

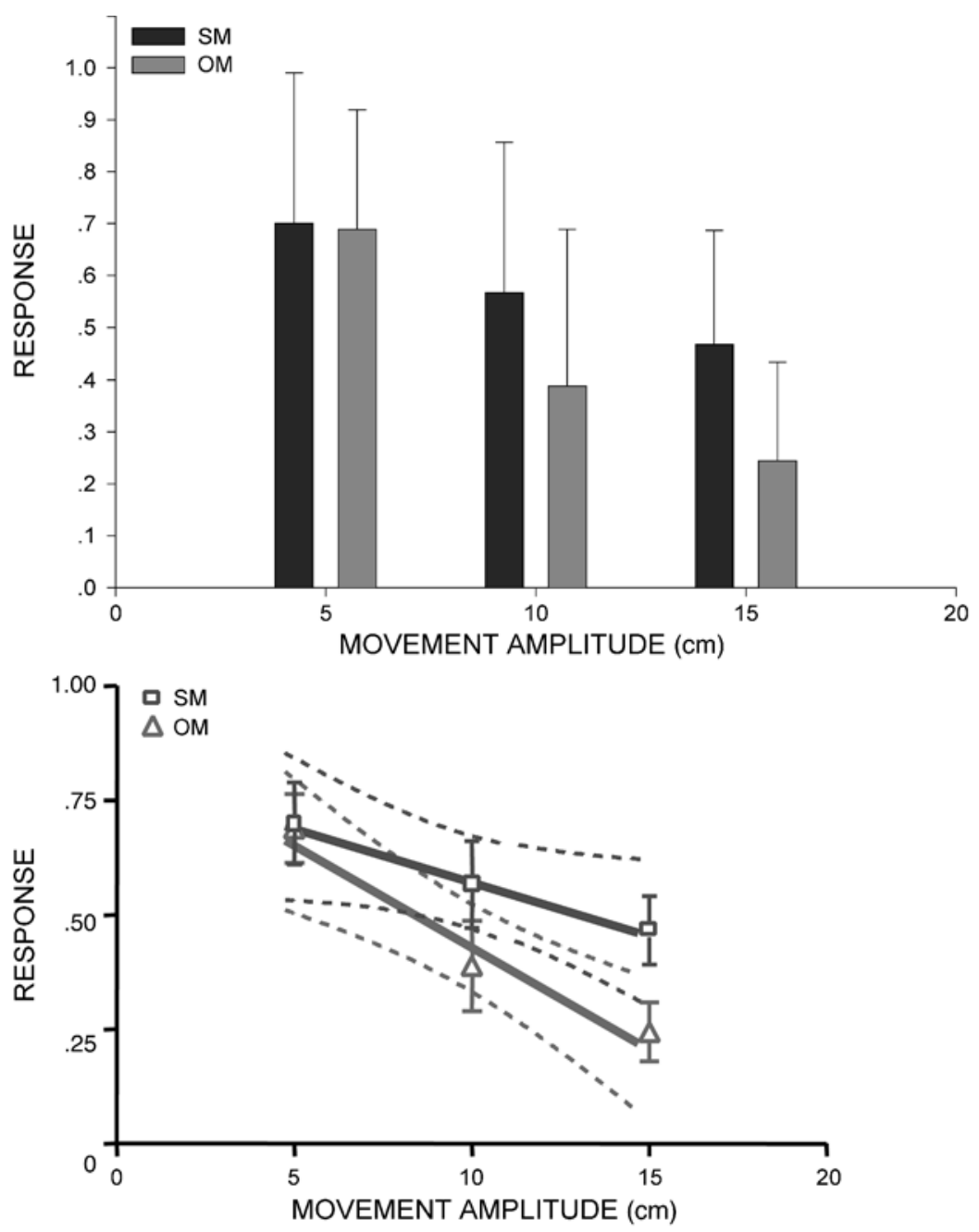

Figure 11. Subjects' responses for constant object distance. Top: SM and OM responses are plotted as a function of the amplitude of movement. Bottom: linear interpolations of SM and OM responses. Dashed lines represent confidence intervals. In the $O M$ case, the slope is significantly different from zero $[F(1,25)=15.26, p<.05]$.

vealed a significant deviation from zero of the slope of the line interpolating the OM responses, but a nonsignificant one in the SM case (see Table 4 for details of the regression parameters). A significant effect of condition was found for the largest movement amplitudes using a Wilcoxon test $(15 \mathrm{~cm}, p<.05)$.

In Figure 11, the reduction in the OM proportion correct responses with greater movement amplitudes indicates once again that the subjects in the OM condition estimated the distance using the apparent motion of the object: For larger apparent motions, the object was perceived as closer. The only important result, however, is the different dependence on movement amplitude found in the SM and OM conditions. This supports our hypothesis of a scaling of visual motion cues by the extraretinal cues. Finally, note that for the smaller amplitude $(5 \mathrm{~cm})$, the $\mathrm{SM}$ and $\mathrm{OM}$ conditions yielded similar responses. This observation is compatible with the fact

Table 4

OM and SM Responses: Regression Parameters

\begin{tabular}{ccccc}
\hline $\begin{array}{c}\text { Viewing } \\
\text { Condition }\end{array}$ & Slope & 95\% Confidence Interval & Deviation From Zero & \multicolumn{1}{c}{$F$ Test } \\
\hline OM & $-0.04 \pm 0.01$ & -0.067 to -0.021 & Significant & $F(1,25)=15.26, p<.05$ \\
SM & $-0.02 \pm 0.01$ & -0.048 to 0.001 & Not significant & $F(1,25)=3.73, p>.05$ \\
\hline
\end{tabular}


that, as the amplitude decreases, the SM and OM conditions are closer to each other since extraretinal information becomes negligible if the amplitude of movement tends toward zero.

\section{GENERAL DISCUSSION}

The main purpose of these experiments was to test the hypothesis that extraretinal signals elicited during selfmotion contribute to scale motion-parallax information. In Experiment 3, judgments of distance were compared, as given by moving (SM) and by stationary (OM) observers receiving the same optical stimulation. The subjects performing in the OM condition could not discriminate distance correctly. This is shown in Figure 10, left, where the proportion correct responses do not correlate with the distance of the objects, but rather indicate chance level. This result is not surprising and accounts for the fact that stimuli for the three different distances were optically identical. However, note that in the SM condition, although the optic array presented identical stimulation, the subjects estimated distance correctly. Our interpretation is that the failure in the OM condition was due to the absence of extraretinal signals. Furthermore, it is worth noting that the subjects had no prior knowledge of the three-dimensional movement in the OM condition, because the movement amplitude was different in each successive trial. This was not the case in Experiment 2, in which the subjects performed head movements of constant amplitude, a method commonly used in many studies of absolute distance perception (see, e.g., Gogel \& Tietz, 1979; Johansson, 1973; S. Rogers \& B. J. Rogers, 1992). Therefore, the results of Experiment 3 strongly support the view that, during self-motion, the brain exploits extraretinal signals to calibrate motion parallax to recover absolute distance information. Possibly, an independent mechanism exists that enables the moving observer's visual system to estimate absolute distances. We speculate that for the moving observer, this mechanism could be combined in principle with the other two purely visual mechanisms, providing absolute distance information, such as the use of vertical disparities in binocular vision (B. J. Rogers \& Bradshaw, 1993) and the measurement of the height in the visual field from the horizon or from the ground surface (Sedgwick, 1986).

In Experiment 2 we observed that (1) in both the SM and OM conditions, the subjects' perceived distance was significantly correlated with simulated distance, and (2) distance perceived in the OM condition was affected by a significant systematic underestimation when compared with the SM condition. The first finding was somewhat unexpected. In fact, in the OM condition, the subjects did not have direct access to information on the object's three-dimensional movement and, from a theoretical point of view, the distance from optic flow analysis could only be recovered when the parameters of movement were known (Lee, 1980). Therefore, how could the subjects correctly recover the distance? Two interpretations seem plausible: (1) cognitive factors such as memory of the previously executed movement (i.e., the movement executed in the SM condition) had an influence, or (2) the subjects used a heuristic approach and assumed a constant 3-D movement of the object to derive the distance from its apparent motion. There are studies that show that the dynamics of movement are stored and recalled if subjects are asked to reproduce these movements (Berthoz, Israel, Georges-François, Grasso, \& Tsuzuku, 1995). This being the case, it would be interesting to compare distance responses of subjects under both conditions (SM and $\mathrm{OM}$ ) with responses of subjects under only the $\mathrm{OM}$ condition. As to the second interpretation, earlier studies on slant discrimination from optic flow (Cornilleau-Pérès, Wong, Cheong, \& Droulez, 2000; Domini \& Caudek, 1999) have shown that subjects tend to adopt heuristics. For example, Domini and Caudek showed that the perceptual system of a nonmoving observer interprets first-order components of optic flow (i.e., deformation) in a heuristic manner, inappropriately recovering the 3-D parameter (i.e., slant) defining the attitude of the simulated surface.

As to the second observation of Experiment 2, we discuss it in the context of a simple computational model. The model is derived from the Longuet-Higgins and Prazdny equations (Prazdny, 1983; see the Appendix for the complete derivation of this model) and relates the estimated distance and the real distance when the estimate of self-movement and of visual motion are supposed to be affected by intrinsic errors. By defining with $D$ and $D_{\text {est }}$, respectively, as the real and the estimated distances, with $A$ being the amplitude of movement, $T$ being a normalized visual motion field, and $\varepsilon_{1}$ and $\varepsilon_{2}$ being the errors on the estimation of the egomotion and the retinal visual motion, we can write

$$
\begin{gathered}
D_{\text {est }}=D+\varepsilon_{1} \frac{D}{A}-\varepsilon_{2} \frac{D^{2}}{A T} \\
\sigma^{2}\left(D_{\text {est }}\right)=\left|\varepsilon_{1}\right|^{2}\left(\frac{D}{A}\right)^{2}+\left|\varepsilon_{2}\right|^{2}\left(\frac{D^{2}}{A T}\right)^{2} .
\end{gathered}
$$

Note that in both expressions, the first error term $\left(\varepsilon_{1}\right)$ is linearly dependent on the distance, whereas the second term $\left(\varepsilon_{2}\right)$ is quadratically dependent on the distance. According to Equations 1 and 2, if $\varepsilon_{2}$ is small with respect to $\varepsilon_{1}$ (i.e., $\varepsilon_{2}<<\varepsilon_{1}$ ), the model predicts an error term in the distance estimate and its variability, which increases linearly with the real distance $D$ (recall that the slope of the psychometric curves-the variability of perceived distance-is proportional to the standard deviation of estimated distance). Conversely, if $\varepsilon_{2}$ is large with respect to $\varepsilon_{1}\left(\varepsilon_{2} \gg \varepsilon_{1}\right)$, the model predicts that the error component will increase quadratically with real distance $D$.

In Experiment 2, we found that the subjects performed a significant underestimation of distance in the OM condition with respect to the SM condition. For example, 
note that at $200 \mathrm{~cm}$ the underestimation error in the $\mathrm{OM}$ condition was close to $25 \%$ of the simulated distance value. This result could be explained by Equation 1 of the model if we assume that $\varepsilon_{2}<<\varepsilon_{1}$ (i.e., a larger error associated with the self-motion estimation process with respect to the visual-motion estimation process) and that the brain performs two different intrinsic errors in the two different experimental conditions, OM and SM: $\left(\varepsilon_{1}{ }^{\mathrm{OM}}\right)$ and $\left(\varepsilon_{1}^{\mathrm{SM}}\right)$. Such a difference $\left(\varepsilon_{1}^{\mathrm{SM}}-\varepsilon_{1}\right.$ OM , with $\left.\varepsilon_{1}^{\mathrm{SM}}<\varepsilon_{1}{ }^{\mathrm{OM}}\right)$ could possibly account for the observed underestimation of the OM, compared with the SM responses (see Figure 8). Finally, in both Experiments 1 and 2, we found a significant linear increase of the variability of perceived distance with simulated distance. Our model predicts this behavior if, in Equation 2, we make the assumption that $\varepsilon_{2}<<\varepsilon_{1}$. On the other hand, in regard to perceived distance (Figure 5, left), we are not able to conclude this because the model predicts either an underestimation or an overestimation, depending on the signs of the two error terms, $\varepsilon_{1}$ and $\varepsilon_{2}$. In conclusion, our model explains the results if we assume, first, that the brain performs larger errors in the estimation of selfmotion rather than in visual motion, and second, that the error associated with self-motion estimation, $\varepsilon_{1} \mathrm{SM}$, is smaller than the error associated with the estimation of the object 3-D movement, $\varepsilon_{1}$ OM, whether object movement is assumed or is recalled from previous self-motion experience.

Two general concerns about the present investigation could be raised. First, the experimental conditions (i.e., screen distance about $60 \mathrm{~cm}$ ) allowed for flatness information from accommodation to be present. We shall argue that flatness cues from accommodation could have played a role in reducing object depth (i.e., relative) but should not have impacted the results according to the task definition (i.e., the subject had to estimate the distance of the more proximal surface of the virtual object). On the other hand, it is not possible to exclude a slight bias in the distance estimates, present for all simulated distances. Logically, this bias would be constant and spread over the whole simulated distance range. In conclusion, from the overall results obtained from the three experiments, it seems unlikely that flatness cues from accommodation could have played a significant role. Second, although in all the experiments the room was set to nearly complete darkness (except for the visual stimulation, which was at low brightness level, see the Method section), one cannot exclude that some subjects might have been able to detect barely visible peripheral contrasts. How effectual could this visual information have been? Even if unattended, such peripheral contrast might have contributed to the subjects' self-motion perception. However, this contribution was very likely weak with respect to vestibular, proprioceptive, and active cues. As for the respective contribution of these cues to the superior performance observed in the SM condition, it remains to be determined in further experiments.
Two additional results emerged from Experiment 1. The first was that the variability of the perceived distance appeared to be slightly reduced when objects were superimposed onto a horizontal ground floor. Such a finding is in accordance with previous studies, for example, Kunnapas (1968), who showed that if the number of cues increases, the accuracy of the depth percept increases too. Furthermore, J. J. Gibson's (1950) ground theory predicts larger uncertainties in absolute distance judgments in the case of an absent or disrupted common ground surface (Sinai et al., 1998). The second result concerns the amplitude of the stimulated visual field. In our framework, field amplitude had no influence whatsoever, either on precision or on the accuracy of the perceived distance. On the contrary, according to previous studies, large-field stimulation seems to improve the estimation of three-dimensional parameters (Dijkstra et al., 1995). Finally, as for the statistical analysis performed on the head trajectories in Experiment 1, recall that the ANOVA conducted on the amplitude of head movement revealed (1) that the head movement was not influenced by the presence of a horizontal ground surface; this result corroborates the finding of a decreased variability of perceived distance in the presence of a ground floor and (2) a significant distance $X$ angular size interaction. This interaction could likely explain the significant distance $X$ angular size interaction found with the ANOVA on the bias parameter of the subject's psychometric curves.

The exact nature of the extraretinal signals still remains to be determined. When active movements of the head or body take place, sensory cues from the vestibular and proprioceptive systems simultaneously converge on the central nervous system. Besides these two components, the efference copies of motor commands are also elicited as a consequence of any voluntary action. The question of whether active control or proprioception (or both) contributes to the superior performance obtained in the self-motion condition is still to be experimentally addressed. On the same line of discussion, one could ask whether our results are specifically valid for active head movements or whether they could be generalized to other types of active controls. In fact, it is possible that in the self-motion condition of our experiments, both types of sensory information were integrated with the efference copy of motor commands in order to obtain a more robust estimate of head movement. Recent findings tend to support this view, suggesting that neck proprioceptive signals might play an important role in providing an independent estimate of head movement, in addition to vestibular signals (Gdowski, Boyle, \& McCrea, 2000; Gdowski \& McCrea, 2000).

\section{REFERENCES}

Berthoz, A., Israel, I., Georges-François, P., Grasso, R., \& TsuzuKu, T. (1995). Spatial memory of body linear displacement: What is being stored? Science, 269, 95-98.

Bingham, G. P., \& Stassen, M. G. (1994). Monocular egocentric dis- 
tance information generated by head movement. Ecological Psychology, 6, 219-238.

Bourdon, B. (1898). La perception monoculaire de la profondeur [Monocular perception of depth]. Revue Philosophique, 46, 124-145.

Bourdon, B. (1902). La perception visuelle de l'espace [Visual perception of space]. Paris: Schleicher Frères.

Braunstein, M. L. (1976). Depth perception through motion. New York: Academic Press.

Braunstein, M. L., Andersen, G. J., Rouse, M. W., \& Tittle, J. S. (1986). Recovering viewer-centered depth from disparity, occlusion, and velocity gradients. Perception \& Psychophysics, 40, 216-224.

Bruno, N., \& Cutting, J. E. (1988). Minimodularity and the perception of layout. Journal of Experimental Psychology: General, 117, 161-170.

Clark, J. J., \& Yuille, A. L. (1990). Data fusion for sensory information processing systems. Boston: Kluwer.

Coello, Y., \& Grealy, M. A. (1997). Effect of size and frame of visual field on the accuracy of an aiming movement. Perception, 26, 287-300.

Collett, T. S. (1978). Peering: A locust behavior pattern for obtaining motion parallax information. Journal of Experimental Biology, 76, 237-241.

Collett, T. S., \& Harkness, L. I. K. (1982). Depth vision in animals. In D. I. Ingle, M. A. Goodale, \& R. J. W. Mansfield (Eds.), Analysis of visual behavior (pp. 111-176). Cambridge, MA: MIT Press.

Cornilleau-Pérès, V., \& Droulez, J. (1994). The visual perception of three-dimensional shape from self-motion and object-motion. $\mathrm{Vi}$ sion Research, 34, 2331-2336.

Cornilleau-Pérès, V., Wong, T. K., Cheong, L. F., \& Droulez, J. (2000). Visual perception of slant from optic flow under orthographic and perspective projection. Investigative Ophthalmology \& Visual Science, 41, 3820.

Cutting, J. E., \& Vishton, P. M. (1995). Perceiving layout and knowing distances: The integration, relative potency, and contextual use of different information about depth. In W. Epstein \& S. Rogers (Eds.), Perception of space and motion (pp. 69-117). San Diego: Academic Press.

DEES, J. W. (1966). Accuracy of absolute visual distance and size estimation in space as a function of stereopsis and motion parallax. Journal of Experimental Psychology, 72, 466-476.

Dichgans, J., \& BRANDT, T. (1978). Visual-vestibular interaction: Effects on self-motion perception and postural control. In R. Held, H. W. Leibowitz, \& H.-L. Teuber (Eds.), Handbook of sensory physiology: Perception (pp. 756-804). Berlin: Springer-Verlag.

Dijkstra, T. M., Cornilleau-Pérès, V., Gielen, C. C., \& Droulez, J. (1995). Perception of three-dimensional shape from ego- and objectmotion: Comparison between small- and large-field stimuli. Vision Research, 35, 453-462.

Domini, F., \& CAUdeK, C. (1999). Perceiving surface slant from deformation of optic flow. Journal of Experimental Psychology: Human Perception \& Performance, 25, 426-444.

Ellard, C. G., Goodale, M. A., Maclaren Scorfield, D. M., \& LAWRENCE, C. (1986). Visual cortical lesions abolish the use of motion parallax in the Mongolian gerbil. Experimental Brain Research, 64, 599-602.

Ellard, C. G., Goodale, M. A., \& Timney, B. (1984). Distance estimation in the Mongolian gerbil: The role of dynamic depth cues. $B e$ havioural Brain Research, 14, 29-39.

ERIKSSON, E. S. (1973). Distance perception and the ambiguity of visual stimulation: A theoretical note. Perception \& Psychophysics, 13, 379-381.

ERIKSSON, E. S. (1974). Movement parallax during locomotion. Perception \& Psychophysics, 16, 197-200.

FERRIS, S. H. (1972). Motion parallax and absolute distance. Journal of Experimental Psychology, 95, 258-263.

Fisher, S. K., \& CiUfFredA, K. J. (1988). Accommodation and apparent distance. Perception, 17, 609-621.

Foster, D. H., \& BischoF, W. F. (1997). Bootstrap estimates of the statistical accuracy of thresholds obtained from psychometric functions. Spatial Vision, 11, 135-139.

Gdowski, G. T., Boyle, R., \& McCrea, R. A. (2000). Sensory processing in the vestibular nuclei during active head movements. Archives Italiennes de Biologie, 138, 15-28.
GDowski, G. T., \& McCreA, R. A. (2000). Neck proprioceptive inputs to primate vestibular nucleus neurons. Experimental Brain Research, 135, 511-526.

Gibson, E. J., Gibson, J. J., Smith, O. W., \& Flock, H. (1959). Motion parallax as a determinant of perceived depth. Journal of Experimental Psychology, 58, 40-51.

GiBson, J. J. (1950). The perception of the visual world. Boston: Houghton Mifflin.

Gogel, W. C. (1961). Convergence as a cue to absolute distance. Journal of Psychology, 52, 287-301.

Gogel, W. C. (1982). Analysis of the perception of motion concomitant with a lateral motion of the head. Perception \& Psychophysics, 32, 241-250.

Gogel, W. C., \& Tietz, J. D. (1973). Absolute motion parallax and the specif ic distance tendency. Perception \& Psychophysics, 13, 284-292.

Gogel, W. C., \& TiETZ, J. D. (1979). A comparison of oculomotor and motion parallax cues of egocentric distance. Vision Research, 19, $1161-1170$

Gonzalez, E. G., Steinbach, M. J., Ono, H., \& Wolf, M. E. (1989). Depth perception in children enucleated at an early age. Clinical Vision Science, 4, 173-177.

Goodale, M. A., Ellard, C. G., \& Booth, L. (1990). The role of image size and retinal motion in the computation of absolute distance by the Mongolian gerbil (Meriones unguiculatus). Vision Research, 30, 399-413.

GRANT, V. W. (1942). Accommodation and convergence in visual space perception. Journal of Experimental Psychology, 31, 89-104.

Johansson, G. (1973). Monocular movement parallax and near-space perception. Perception, 2, 135-146.

KoENDERINK, J. J. (1986). Optic flow. Vision Research, 26, 161-180.

KoENDERINK, J. J., \& VAN DoORN, A. J. (1975). Invariant properties of the motion parallax field due to the movement of rigid bodies relative to an observer. Optica Acta, 22, 773-791.

KunNaPAS, T. (1968). Distance perception as a function of available visual cues. Journal of Experimental Psychology, 77, 523-529.

Landy, M. S., Maloney, L. T., Johnston, E. B., \& Young, M. (1995). Measurement and modeling of depth cue combination: In defense of weak fusion. Vision Research, 35, 389-412.

LEe, D. N. (1980). The optic flow field. Philosophical Transactions of the Royal Society of London, Series B, 290, 169-179.

Marotta, J. J., Kruyer, A., \& Goodale, M. A. (1998). The role of head movements in the control of manual prehension. Experimental Brain Research, 120, 134-138.

Marotta, J. J., Perrot, T. S., Nicolle, D., Servos, P., \& Goodale, M. A. (1995). Adapting to monocular vision: Grasping with one eye. Experimental Brain Research, 104, 107-114.

Pagano, C. C., \& Bingham, G. P. (1998). Comparing measures of monocular distance perception: Verbal and reaching errors are not correlated. Journal of Experimental Psychology: Human Perception \& Performance, 24, 1037-1051.

Palmer, S. E. (1999). Vision science: Photons to phenomenology. Cambridge, MA: MIT Press.

Panerai, F., Hanneton, S., Droulez, J., \& Cornilleau-Pérès, V. (1999). A 6-dof device to measure head movements in active vision experiments: Geometric modeling and metric accuracy. Journal of Neuroscience Methods, 90, 97-106.

PARK, J. N. (1964). The role of motion parallax in depth perception. Unpublished doctoral dissertation, University of Kansas, Lawrence.

Philbeck, J. W., Loomis, J. M., \& Beall, A. C. (1997). Visually perceived location is an invariant in the control of action. Perception \& Psychophysics, 59, 601-612.

Poom, L., \& BöRJESson, E. (1999). Perceptual depth synthesis in the visual system as revealed by selective adaptation. Journal of Experimental Psychology: Human Perception \& Performance, 25, 504-517.

Prazdny, K. (1983). Information in optic flows. Computer Vision, Graphics, \& Image Processing, 22, 235-259.

Rogers, B. [J.], \& Graham, M. (1979). Motion parallax as an independent cue for depth perception. Perception, 8, 125-134.

Rogers, B. J., \& BradshaW, M. F. (1993). Vertical disparities, differential perspectives and binocular stereopsis. Nature, 361, 253-255.

Rogers, S., \& Rogers, B. J. (1992). Visual and nonvisual information 
disambiguate surfaces specified by motion parallax. Perception \& Psychophysics, 52, 446-452.

SEDGWICK, H. A. (1986). Handbook of perception and human performance: Sensory processes and perception. New York: Wiley.

Simpson, W. A. (1993). Optic flow and depth perception. Spatial Vision, 7, 35-75.

SinaI, M. J., OoI, T. L., \& He, Z J. (1998). Terrain influences the accurate judgment of distance. Nature, 395, 497-500.

Steinbach, M. J., Ono, H., \& Wolf, M. E. (1991). Motion parallax judgments of depth as a function of the direction and type of head movement. Canadian Journal of Psychology, 45, 92-98.

van Damme, W. J., \& VAN DE GRIND, W. A. (1996). Non-visual information in structure-from-motion. Vision Research, 36, 3119-3127.

WAllach,H., \& Floor, L. (1971). The use of size matching to demon- strate the effectiveness of accommodation and convergence as cues for distance. Perception \& Psychophysics, 10, 423-428.

Wexler, M., Panerai, F., Lamouret, I., \& Droulez, J. (2001). Selfmotion and the perception of stationary objects. Nature, 409, $85-$ 88.

Wickelgren, E. A., McConnell, D. S., \& Bingham, G. P. (2000). Reaching measures of monocular distance perception: Forward versus side-to-side head movements and haptic feedback. Perception \& Psychophysics, 62, 1051-1059.

\section{NOTE}

1. Optic flow is the pattern of motion on the retina (J. J. Gibson, 1950).

\section{APPENDIX}

Here we develop a simple model aimed at describing the relationship between the estimated distance $D_{\text {est }}$ and the real distance $D$ as a function of the errors that we assume to be present in the sensory measurements of the 3-D visual motion. Starting from the equations of Longuet-Higgins and Prazdny (Prazdny, 1983), we derived the following simplified version for the translational component:

$$
U(x, y)=\frac{[A] \cdot T(x, y)}{D},
$$

where $U(x, y)$ is the horizontal distribution of 2-D velocities for every point in the retina, $T(x, y)$ is a normalized 3 -D velocity field, and $A$ is the amplitude of the 3-D movement. If we suppose that the brain estimates the movement $A$ with an intrinsic error $\varepsilon_{1}\left(A_{\text {est }}=A+\varepsilon_{1}\right)$ and measures the retinal motion with an intrinsic error $\varepsilon_{2}$, we can rewrite Equation A1, which is the exact formulation derived from Longuet-Higgins, as follows:

$$
\begin{gathered}
D_{\text {est }}=\frac{\left[A_{\text {est }}\right] \cdot T(x, y)}{U(x, y)+\varepsilon_{2}} \\
D_{\text {est }}=\frac{\left[A+\varepsilon_{1}\right] \cdot T}{A T / D+\varepsilon_{2}}
\end{gathered}
$$

$$
D_{\text {est }}=\frac{D\left[A+\varepsilon_{1}\right] \cdot\left[1-\varepsilon_{2} D / A T\right]}{A \cdot\left[1+\varepsilon_{2} D / A T\right] \cdot\left[1-\varepsilon_{2} D / A T\right]} .
$$

Because $\varepsilon_{2}<<A T / D$, the previous denominator can be approximated by the following:

$$
\begin{gathered}
D_{\text {est }} \cong \frac{D\left[A+\varepsilon_{1}\right] \cdot\left[1-\varepsilon_{2} D / A T\right]}{A} \\
D_{\text {est }} \cong D\left[1+\varepsilon_{1} / A\right] \cdot\left[1-\varepsilon_{2} D / A T\right] \\
D_{\text {est }} \cong D+\varepsilon_{1} \frac{D}{A}-\varepsilon_{2} \frac{D_{2}}{A T}-\varepsilon_{1} \varepsilon_{2} \frac{D_{2}}{A_{2} T} .
\end{gathered}
$$

If $\varepsilon_{1} / A$ and $\varepsilon_{2} D / A T=\varepsilon_{2} / U$ are supposed to be much smaller than 1 (because $\varepsilon_{1}<A$ and $\varepsilon_{2}<U$ ) and the noise term is supposed to be Gaussian and have a zero average, it follows that the firstorder estimates of $D_{\text {est }}$ and $\sigma^{2}\left(D_{\text {est }}\right)$ are:

$$
\begin{gathered}
D_{\text {est }}=D+\varepsilon_{1} \frac{D}{A}-\varepsilon_{2} \frac{D^{2}}{A T} \\
\sigma^{2}\left(D_{\text {est }}\right)=\left|\varepsilon_{1}\right|^{2}\left(\frac{D}{A}\right)^{2}+\left|\varepsilon_{2}\right|^{2}\left(\frac{D^{2}}{A T}\right)^{2} .
\end{gathered}
$$

